\title{
The influence of eye-gaze and arrow pointing distractor cues on voluntary eye movements
}

\author{
GuSTAV KuHN \\ University of Durham, Durham, England \\ AND \\ VALERIE BENSON \\ University of Southampton, Southampton, England
}

\begin{abstract}
We investigated Ricciardelli et al.'s (2002) claim, that the tendency for gaze direction to elicit automatic attentional following is unique to biologically significant information. Participants made voluntary saccades to targets on the left or the right of a display, which were either congruent or incongruent with a centrally presented distractor (eye-gaze or arrow). Contrary to Ricciardelli et al., for both distractor types, saccade latencies were slower, and participants made more directional errors, on incongruent than on congruent trials. Moreover, a cost-benefit analysis showed no difference between the two distractor types. However, latencies for erroneous saccades were faster than correctly directed saccades for the eye-gaze distractors, but not for the arrow distractors.
\end{abstract}

There is growing evidence demonstrating that another person's gaze direction can lead to automatic shifts in attention toward the fixated location, a phenomenon known as socially directed attention. Using a variation of Posner's (1980) precuing paradigm it has been shown that spatially nonpredictive gaze direction cues facilitate reaction times to targets appearing at the fixated location (e.g., Friesen \& Kingstone, 1998). These effects have been found even when the gaze direction was counterpredictive to the target location (e.g., Driver, Davies, \& Ricciardelli, 1999), which has led to the suggestion that such shifts in attention are obligatory and occur automatically.

Similar findings have been reported with overt attention (gaze shifts). Ricciardelli et al. (2002) showed that people follow another's gaze direction even when the task does not demand this. In their study, observers were required to make a voluntary (goal directed) saccade toward a peripheral target. An image of a real face was presented in the center of the display with eye gaze directed either toward the target location (congruent), or in the opposite direction to it (incongruent). Ricciardelli et al. showed that participants' saccade latencies (the time from the onset of the display to the initiation of the first saccade in the direction of the target) were significantly faster for congruent compared to incongruent trials. This effect was also found when the face was replaced by nonpredictive arrows. Furthermore, participants made more errors on incongruent than on congruent trials. Most importantly, although the trend was in the predicted direction this latter effect was not significant in a condition where the face was replaced by nonpredictive arrows. The authors took this finding to suggest that automatic gaze imitation behavior was unique to biologically relevant stimuli.

Ricciardelli et al.'s (2002) claim is consistent with the traditional view that arrow cues only lead to shifts in attention if the arrow is predictive of the target location (see Kingstone, Smilek, Ristic, Friesen, \& Eastwood, 2003). However, more recently it has been shown that nonpredictive arrows can lead to shifts in covert attention (Ristic, Friesen, \& Kingstone, 2002; Tipples, 2002). This suggests that symbolic cues can trigger automatic shifts of attention. The failure to obtain such shifts in the experiment of Ricciardelli et al. (2002) might be because of the nature of the stimuli they used. Ricciardelli et al. (2002) created arrows by changing Xs into "less-than" or "greater-than" symbols $(<<$ or $>>)$ pointing either left or right, which are rather different from arrows used in the "real world" and from those used in most other social attention experiments (e.g., Ristic et al., 2002; Tipples, 2002), and it is therefore possible that Ricciardelli et al. found no cuing effects with these types of arrows because of their "unarrow-like" properties rather than their symbolic nature. Moreover, Ricciardelli et al. did not directly compare the effectiveness of the arrow and the eye-gaze cues.

In the following experiments we investigated participants' gaze following by adopting Ricciardelli et al.'s (2002) paradigm, but using arrows that appeared to be more "arrow-like" than theirs. Our design allowed us to directly compare the effectiveness of biologically relevant and symbolic cues. If gaze following is unique to biologically relevant stimuli, the eye-gaze cues should lead to greater interference on participants' saccades

G.Kuhn, gustav.kuhn@durham.ac.uk 
than do the arrow cues, regardless of how salient the arrow cues are.

\section{EXPERIMENT 1}

\section{Method}

Participants. Eight University of Durham undergraduate students ( 6 female, 2 male) were paid (£5) to participate in the study. All participants had normal, uncorrected vision, and were naive to the purpose of the experiment.

Eye movement recording. Eye movements were recorded using a Fourward Technologies Dual Purkinje Generation 5.5 eye tracker with spatial resolution of $10 \mathrm{~min}$ of arc. Viewing was binocular, but the movements of only one eye were monitored. Stimulus files were displayed on a Phillips 21B582BH 21-in. monitor at a viewing distance of $1 \mathrm{~m}$. The monitor had a P22 phosphor with a decay rate to zero of less than $2 \mathrm{msec}$. The monitor and the eyetracker were both interfaced with a Phillips Pentium III PC that controlled the experiment. Eye position was sampled every $5 \mathrm{msec}$.

Materials and Procedure. Two types of stimulus files were created, one for the eye-gaze and one for the arrow condition. Each of the stimulus files displayed two possible targets, one positioned to the left and one to the right of the midline of the display, each at an eccentricity of $8^{\circ}$ from the center of the screen. Targets were solid black circles subtending approximately $1^{\circ}$ of visual angle, and the background of each display was gray. In the eye-gaze condition the sequence of presentation for each trial was as follows (see Figure 1): a schematic face with the eyes looking straight ahead was presented centrally, flanked by the two solid black target circles. Between the eyes there was a small black spot that acted as a fixation point. After $1,000 \mathrm{msec}$, the fixation point changed color to either green or red, and simultaneous with this, the eye-gaze of the schematic face changed to look to the left or the right. Half of the participants were instructed to move their eyes to the right when the fixation point turned red and to the left when it turned green, and for the other half of the participants the color commands were reversed. The color change of the fixation spot therefore informed each participant as to which target they had to saccade to. Congruent trials were those where the saccade target direction and the eye gaze direction were the same; incongruent trials were conditions in which the target and the eye gaze direction were opposite to each other. After 1,000 msec, the display was replaced with a blank screen for $1,500 \mathrm{msec}$.

Eight participants were presented with examples of the arrows used in the present study and arrows used by Ricciardelli et al. (2002) and were asked to rate on a 10 point Likert scale how much they though the arrow resembled a prototypical arrow. Participants rated our arrows $(M=8.0, S D=1)$ significantly higher than those used by Ricciardelli et al. $(M=5.7, S D=1.7)[t(7)=3.50, p=.01]$.
In the arrow condition the targets were identical to the eye-gaze condition but the schematic face was replaced with a horizontal line with a black diamond at each end of the line, with the fixation spot at the center of the line. Simultaneously with the fixation point changing color, the right or the left hand sides of the diamonds were removed, thus changing the display into an arrow facing either to the right or the left. As in the previous condition the color of the fixation point indicated the direction in which participants were required to move their eyes. Participants were told to fixate on the central fixation point at the beginning of each trial and saccade to the left or the right hand target, as indicated by the color switch of the fixation point, and to ignore completely the distractor cues. Participants were urged to move their eyes as quickly as possible to the target and to avoid making any mistakes.

Participants were tested in both the arrow and the eye-gaze condition in separate blocks, the order of which was counterbalanced across participants. Each block comprised 100 trials (50 congruent trials and 50 incongruent trials) presented randomly. Prior to each block a total of 20 practice trials were given. The dependent variables were eye movement onset latencies and directional errors. It was predicted that, if eye-gaze following is unique to biologically relevant stimuli, congruent trials should produce shorter latencies than the incongruent trials and that these differences should be absent with the arrow distractors. Moreover, participants should make errors on incongruent trials in the eye-gaze condition, but not with arrow distractors.

\section{Results and Discussion}

For this experiment and Experiment 2, a semiautomated procedure was used to analyze the eye movement data. Eye movement onset latency was measured as time elapsing from the fixation spot color change to the initiation of a saccade. The first saccade was detected automatically using a velocity criterion of approximately $30 \% \mathrm{sec}$ and each record was inspected individually. Any trials in which tracker loss occurred were excluded from the analysis ( $7.06 \%$ of trials). Saccade latencies that were greater or smaller than the mean $\pm 3 S D$ were classified as outliers and were also removed prior to analysis. Figure 2A shows the mean saccade latencies for saccades in the correct direction on congruent and incongruent trials for the arrow and the eye-gaze conditions. A repeated measures ANOVA with distractor type (eyegaze vs. arrow) and validity (congruent vs. incongruent) as within-subjects factors found a significant main effect of validity $[F(1,7)=77.9, p<.0001]$, demonstrating that saccade latencies on congruent trials $(M=344, S E=14.5)$ were significantly shorter than on incongruent trials $(M=$

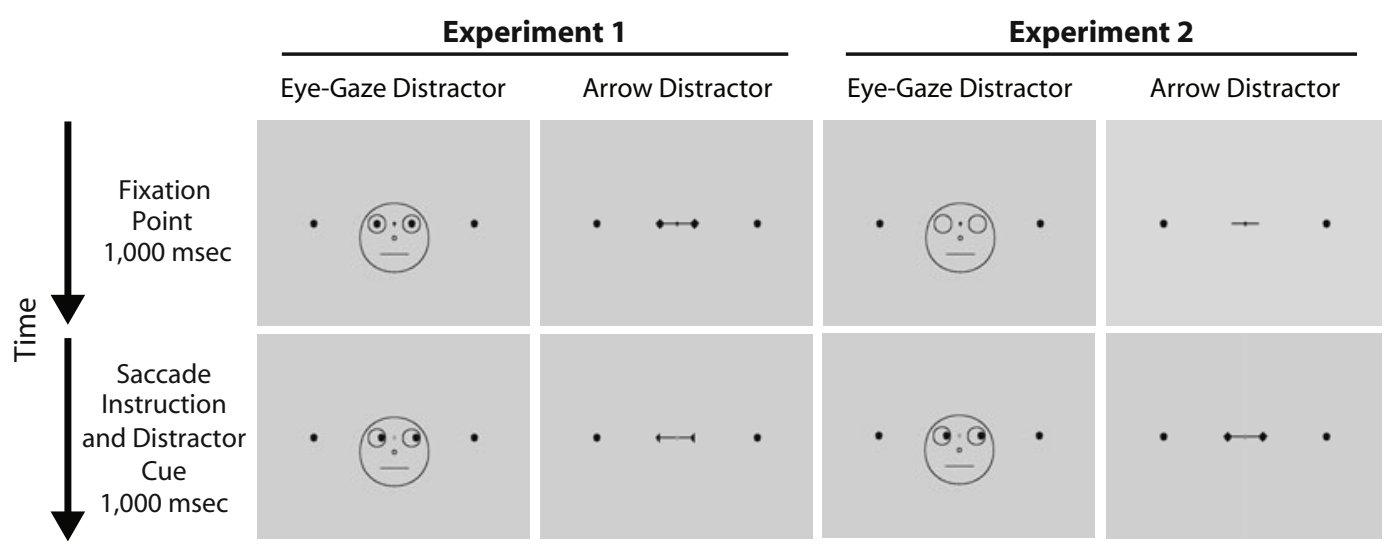

Figure 1. Schematic diagrams of the trial sequence for Experiments 1 and 2. 

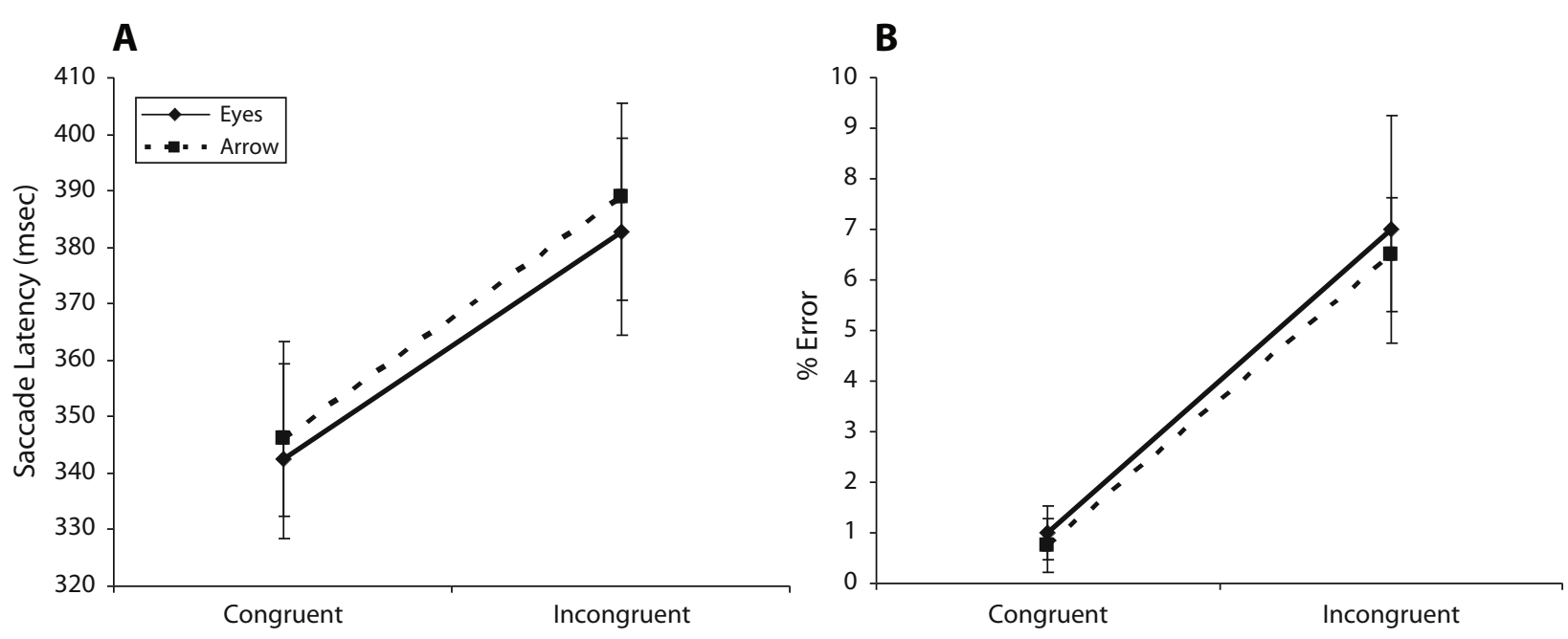

Figure 2. (A) Mean onset latencies for correct saccades to target for congruent and incongruent trials for eye-gaze and arrow distractor conditions in Experiment 1. (B) Mean percentage of directional errors made for congruent and incongruent trials for eye-gaze and arrow distractor conditions in Experiment 1. Error bars denote standard errors.

$385, S E=17.7)$. There was no significant main effect of distractor type $[F(1,7)<1]$, nor was there a significant distractor type by validity interaction $[F(1,7)<1]$, indicating that the congruency effect was of a similar magnitude for the eye-gaze and the arrow distractors.

Errors were trials on which the first saccade was directed to the side of the display opposite to the designated target. If the direction of the distractor cue influences the execution of saccades, we would expect people to make more directional errors on incongruent trials than on congruent trials. Figure 2B shows the percentage of errors for congruent and incongruent conditions for both distractor types. A repeated measures ANOVA with distractor type and validity as within-subjects factor showed a significant main effect of validity $[F(1,7)=18.8, p=.003]$. Participants made more errors on incongruent $(M=6.7 \%, S E=$ 1.35) compared to congruent trials $(M=0.88 \%, S E=$ $0.40)$. There was no significant main effect of distractor type $[F(1,7)<1]$, nor was there any significant interaction $[F(1,7)<1]$ between distractor type and validity. Saccade errors were equally likely to occur for both eye-gaze and arrow distractors.

Experiment 1 showed that participants' voluntary saccades were influenced by nonpredictive eye-gaze and arrow cues, even though they had been explicitly instructed to ignore these. Moreover, the interference found for the arrow distractors was of equal magnitude to that observed for the eye-gaze distractor. The saccade latency data and the directional error data together demonstrate that directional cuing is not unique to biologically relevant stimuli and provide evidence against the claims made by Ricciardelli et al. (2002).

\section{EXPERIMENT 2}

In Experiment 1 we compared saccade latencies and saccade errors between congruent and incongruent trials. We therefore do not know whether the effect of con- gruency resulted from a facilitation on congruent trials, or from a cost on incongruent trials, or both of these. A comparison of saccade latencies between congruent and neutral distractors should provide us with an index of facilitation for congruent trials, while a comparison between incongruent and neutral distractors will provide us with an index of any cost associated with incongruent trials. Using a Posner (1980) type precuing task, Langdon and Smith (2005) showed that unlike eye-gaze cues which trigger facilitation and cost, arrows cues caused facilitation, but not cost. Different studies however do not appear to support this finding. Koval et al. (2005) used a prosaccade task, rather than our voluntary saccade task, in which participants were instructed to make a saccade toward the onset of a target location. In the center of the display there was a picture of a face, whose eye-gaze was either congruent or incongruent with the instructed saccade direction, or looking straight ahead. Saccade latencies on congruent trials were significantly faster than on incongruent and neutral trials, thus demonstrating a significant facilitation effect. However, the difference in saccade latencies for the incongruent and the neutral trials was minimal, thus suggesting that in a prosaccade task eye-gaze leads to facilitation-without-cost, or at least minimal cost.

A further aim of Experiment 2 was to eliminate any confound from apparent motion. In Experiment 1 the eye-gaze cue was achieved by replacing the central pupil in the schematic face with pupils positioned either to the left or the right, and this was noted to create the perception of apparent motion. The arrow distractor cue was created by offsetting either the right or the left hand side of the diamond, and no apparent motion was perceived. In Experiment 2 both distractor cues were created by the onset of stimuli so that neither contained any apparent motion signal.

\section{Method}

Participants. Eight University of Durham undergraduate students ( 3 female, 5 male) were paid $£ 5$ to participate in the experi- 
ment. All participants had normal, uncorrected vision, and were naive to the purpose of the experiment.

Materials and Procedure. The procedure, sequence and timing of display presentation in Experiment 2 were identical to Experiment 1. In the eye-gaze condition each trial began with a display of a schematic face minus pupils in the eyes rather than the pupils looking straight ahead. Simultaneously with the fixation spot color change, the pupils were onset, and these were either looking straight ahead (neutral condition) or directed to the left or the right (see Figure 1). In the arrow condition, each trial began with a display of a horizontal line, and simultaneously with the fixation spot color change either a diamond appeared at each end of the line (neutral condition), or an arrow-head appeared at each end of the line, pointing toward the right or the left. Each participant was tested in both the arrow and the eye-gaze condition, the order of which was counterbalanced. Each block comprised of 150 trials, with three conditions (congruent vs. neutral vs. incongruent), each being equally probable and presented in random order.

\section{Results and Discussion}

The data analysis was the same as for Experiment 1, with the exception of the additional neutral condition. Signal loss trials $(7.9 \%)$ were excluded from the analysis. Figure 3A shows participants' saccade latencies. A repeated measures ANOVA with distractor type (arrow vs. eyes) and validity (congruent vs. neutral vs. incongruent) as within-subjects variables showed a significant main effect of validity $[F(2,7)=6.91, p=.008]$, but no significant main effect of stimulus type $[F(1,7)=1.73, p=.23]$, and no significant interaction $[F(2,7)<1]$, between the two. Similar to Experiment 1 eye-gaze and arrow distractors generated the same pattern of effects on saccade latencies to correct targets. One-tailed Bonferroni corrected pairwise comparisons showed that saccade latencies for congruent trials were significantly faster than neutral $(p=.024)$ and incongruent $(p=.003)$ trials but that saccade latencies for incongruent trials were no different to the neutral trials $(p=.94)$, thus demonstrating that both eye-gaze and arrow distractors resulted in facilitation effects but not cost effects.

Figure 3B shows the percentage of saccade errors for each condition. A repeated measures ANOVA with dis- tractor type (arrow vs. eyes) and validity (congruent vs. neutral vs. incongruent) as within-subjects variables found a significant main effect of validity $[F(1,7)=$ $19.3, p<.0001]$, but no significant main effect of distractor type $[F(2,7)<1]$, and no significant interaction $[F(2,7)<1]$ between the two. Similar to the findings from Experiment 1, arrow distractors and eye-gaze distractors produced an equivalent proportion of directional errors toward the cued direction. One-tailed Bonferroni corrected pairwise comparisons showed that participants made significantly more errors on the incongruent than on the neutral trails ( $p=.015)$, and significantly less errors on the congruent ( $p=.028)$, than on the neutral trials, thus demonstrating both cost and benefit.

The analyses so far have only considered latencies for correct responses. A further exploratory analysis was conducted to investigate the nature of participants' saccades when they incorrectly moved their eyes to the side of the display that the distractor pointed to. We compared the median ${ }^{1}$ saccade latencies for incorrect responses on incongruent trials with the saccade latencies for correct responses on incongruent trials, for both arrow and eyegaze distractor types. In order to gain statistical power, we included the data from both experiments. Data from 5 participants were excluded [ 3 from Experiment 1 ( 3 female, 1 male); and 2 from Experiment 2 (2 male)], since these participants made no errors in one or more of the conditions. The mean saccade latencies for correct and incorrect trials are presented in Table 1. An ANOVA with saccade accuracy (correct vs. incorrect) and distractor type (eyegaze vs. arrows) as within-subjects factors showed no significant main effect of distractor type $[F(1,10)=2.24$, $p=.17]$, or saccade accuracy $[F(1,10)<1]$. However, there was a significant saccade accuracy by distractor type interaction $[F(1,10)=5.21, p=.0046]$. For the eye-gaze distractor trials, erroneous saccades were initiated significantly faster than correct saccades $[t(10)=2.26, p=$ .047], however this difference was not significant for the
A

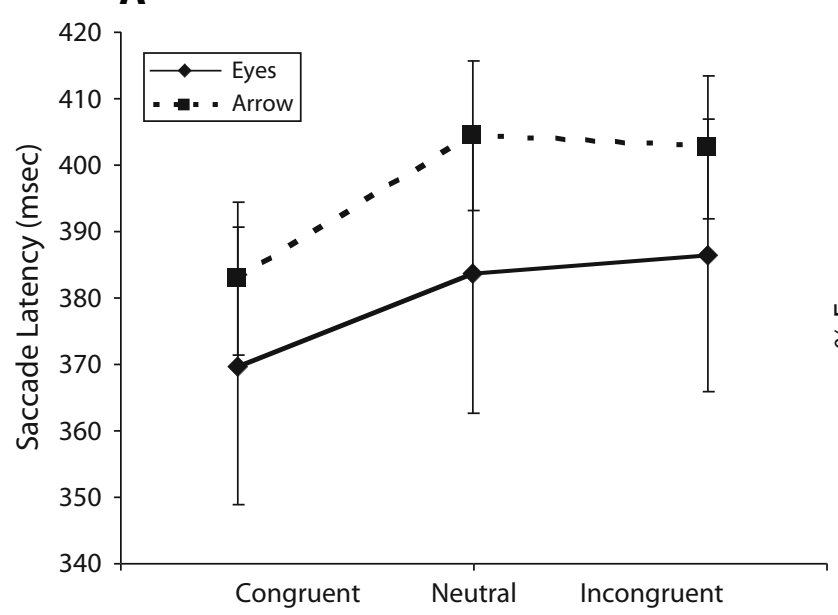

B

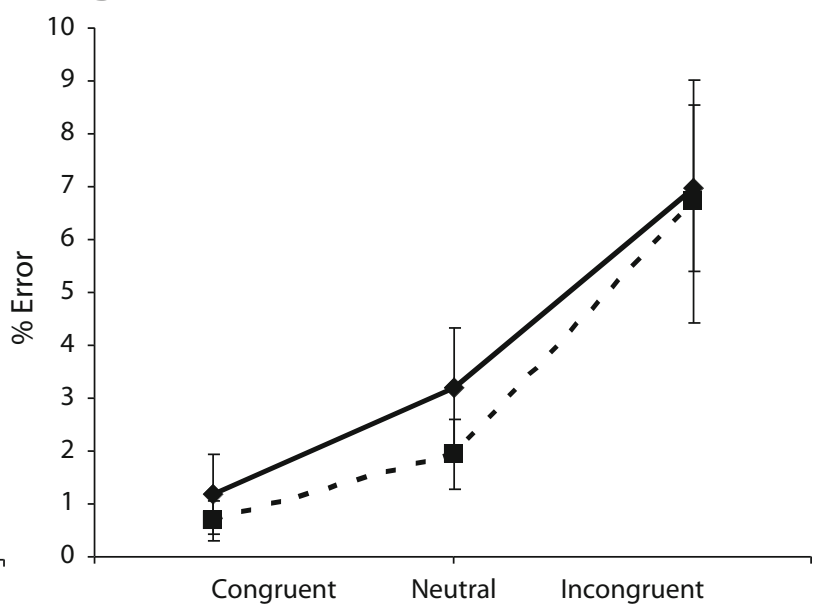

Figure 3. (A) Mean onset latencies for correct saccades to target for congruent incongruent and neutral trials for eye-gaze and arrow distractor conditions in Experiment 2. Error bars denote standard errors. (B) Mean percentage of directional errors made for congruent, incongruent and neutral trials for eye-gaze and arrow distractor conditions in Experiment 2. Error bars denote standard errors. 
arrow distractor trials $[t(10)<1]$, and in fact there was a trend in the opposite direction.

\section{GENERAL DISCUSSION}

The aim of our paper was to investigate whether eyegaze stimuli influenced the execution of voluntary saccades and whether this interference was unique to biologically relevant stimuli. Similar to Ricciardelli et al. (2002) in Experiments 1 and 2 participants' saccade latencies on congruent trials were significantly faster than on incongruent trials for the eye-gaze and arrow condition. Moreover, participants made significantly more errors on the incongruent than on the congruent trials for each type of distractor which illustrates that at least on some of the trials, the distractor cues triggered automatic cue following. Ricciardelli et al. showed that eye-gaze distractors, but not arrows, resulted in automatic gaze following, and therefore argued that gaze following was unique to biologically relevant cues. Contrary to their claims, we have shown that eye-gaze and arrow distractors were equally effective at eliciting saccades in the nonintended direction. It is unlikely that this difference resulted from our use of a schematic face. Hietanen and Leppanen (2003) compared the cuing effects for schematic faces and pictures of real faces, and found no difference between the two. Moreover, our error rates on incongruent trials in Experiment 1 (7\%) and Experiment $2(7 \%)$ were similar to those reported by Ricciardelli et al. (6.3\%), which suggests that our eyegaze distractor was just as effective as theirs. It seems more likely that the disparity resulted from the use of different arrow cues. Ricciardelli et al.'s arrows were created by turning two Xs into "greater than" $(>>)$ ) or "less-than" $(<<)$ symbols, which looked less "arrow like" than ours. It is therefore more likely that their reported dissociation resulted from the lack of salience of the arrows rather than any special effects of the eye-gaze. Also, the error rates reported by Ricciardelli et al. for arrow cues $(3.3 \%)$ were somewhat lower than the ones found here (7\%). We therefore suggest that their findings are more likely to reflect differences in the salience of the directional cues used, rather than any real differences between biologically significant and symbolic cues.

In Experiment 2, we included a neutral condition which allowed us to analyze our data in terms of facilitation and cost effects that our nonpredictive distractors could have had. Langdon and Smith (2005) showed that while eyegaze showed a facilitation-plus-cost pattern, arrow distractors resulted in facilitation-without-cost effects, thus illustrating a dissociation between biologically relevant and

Table 1

Mean Latencies (in Milliseconds) for Correct and Incorrect Saccades, on Incongruent Trials

\begin{tabular}{|c|c|c|c|c|}
\hline & \multicolumn{2}{|c|}{ Correct } & \multicolumn{2}{|c|}{ Incorrect } \\
\hline & $M$ & $\overline{S D}$ & $M$ & $S D$ \\
\hline Eye gaze & 370.9 & 35.0 & 339.8 & 54.4 \\
\hline Arrow & 378.4 & 52.2 & 389.8 & 99.9 \\
\hline
\end{tabular}

Note-Data were collapsed across Experiments 1 and 2. symbolic cues. Our results on the other hand demonstrated that in terms of saccade latencies both the eye-gaze and the arrow distractor resulted in facilitation-without-cost. Participants' error rates on the other hand showed a somewhat different pattern. Error rates for incongruent trials were significantly higher compared with neutral trials, and error rates for neutral trials were marginally higher compared with congruent trials. Most importantly, there was no difference in the error-rate pattern between eye-gaze cues and arrow distractors, thus again supporting our initial finding that basic cue following was just as likely for biologically relevant and symbolic distractor cues.

The results in terms of saccade latencies on trials in which a saccade was made in the correct direction and participants' error rates suggest that there is no difference between eye-gaze and arrow cues. Errors made on incongruent trials, that were later corrected, could represent reflexive saccades that are a result of attentional capture by an incongruent cue (e.g., Theeuwes, Kramer, Hahn, \& Irwin, 1998), while correct saccades to target on incongruent trials are volitional. If the former saccades are reflexive, we would expect the saccade latencies for incorrect saccades to be shorter than those for correct saccades (e.g., Muller \& Rabbitt, 1989). The analysis of the trials on which participants unintentionally followed the distractor cue rather than the saccade instruction revealed an interesting difference between arrows and eye-gaze. In the eye-gaze condition, participants' saccade latencies for erroneous saccades were significantly faster than for correct saccades, thus demonstrating their reflexive nature. However, in the arrow condition this difference was not significant. It is possible that these findings could merely reflect differences in distractor salience for directing attention. However, this interpretation is at odds with the initial finding that both arrow and eye-gaze cues are equally effective in terms of proportion of saccade errors and correctly directed saccade latencies. Our favored interpretation is that eye-gaze distractors may be more likely to result in fast reflexive erroneous saccades compared to the symbolic arrow distractors. These results suggest that although both types of cues are equally effective at triggering the same proportion of saccades in the opposite to the intended direction, the saccades triggered by eye-gaze may be more reflexive in nature. This difference in the reflexive nature of biologically relevant distractors compared to symbolic distractors may be only one of several subtle differences between these two types of cues. As these results are of an exploratory nature further research is required to identify conditions under which these cues behave differently. For example recent findings by Friesen et al. (2004) have shown that although eye-gaze and arrow cues act very similarly if they are nonpredictive, eye-gaze but not arrow cues lead to automatic shifts in attention if the cues are counter predictive of the target location, which coincides with what we have shown here.

\section{AUTHOR NOTE}

The first author was supported by a fellowship from the Wolfson Research Institute at the University of Durham. We thank Simon P. Liversedge and John M. Findlay for their helpful comments. Correspon- 
dence concerning this article should be addressed to G. Kuhn, Department of Psychology, University of Durham, South Road, Durham DH1 3LE, England (e-mail: gustav.kuhn@durham.ac.uk).

\section{REFERENCES}

Driver, J., Davies, M., \& Ricciardelli, P. (1999). Gaze perception triggers reflective visuospatial orienting. Visual Cognition, 6, 509-540.

Friesen, C. K., \& Kingstone, A. (1998). The eyes have it! Reflexive orienting is triggered by nonpredictive gaze. Psychonomic Bulletin \& Review, 5, 490-495.

Friesen, C. K., Ristic, J., \& Kingstone, A. (2004). Attentional effects of counterpredictive gaze and arrow cues. Journal of Experimental Psychology: Human Perception \& Performance, 30, 319-329.

Hietanen, J. K., \& LePpanen, J. M. (2003). Does facial expression affect attention orienting by gaze direction cues? Journal of Experimental Psychology: Human Perception \& Performance, 29, 1228-1243.

Kingstone, A., Smilek, D., Ristic, J., Friesen, C. K., \& Eastwood, J. D. (2003). Attention, researchers! It is time to take a look at the real world. Current Directions in Psychological Science, 12, 176-180.

Koval, M. J., Thomas, B. S., \& Everling, S. (2005). Task-dependent effects of social attention on saccadic reaction times. Experimental Brain Research, 167, 475-480.

LANGDON, R., \& SMith, P. (2005). Spatial cueing by social versus nonsocial directional signals. Visual Cognition, 12, 1497-1527.

Muller, H. J., \& RABbitt, P. M. A. (1989). Reflexive and voluntary orienting of visual-attention: Time course of activation and resistance to interruption. Journal of Experimental Psychology: Human Perception \& Performance, 15, 315-330.

Posner, M. I. (1980). Orienting of attention. Quarterly Journal of Experimental Psychology, 32, 3-25.

Ricciardelli, P., Bricolo, E., Aglioti, S. M., \& Chelazzi, L. (2002). My eyes want to look where your eyes are looking: Exploring the tendency to imitate another individual's gaze. NeuroReport, 13, 2259-2264.

Ristic, J., Friesen, C. K., \& Kingstone, A. (2002). Are eyes special? It depends on how you look at it. Psychonomic Bulletin \& Review, 9, 507-513.

Theeuwes, J., Kramer, A. F., Hahn, S., \& Irwin, D. E. (1998). Our eyes do not always go where we want them to go: Capture of the eyes by new objects. Psychological Science, 9, 379-385.

Tipples, J. (2002). Eye gaze is not unique: Automatic orienting in response to uninformative arrows. Psychonomic Bulletin \& Review, 9, 314-318.

\section{NOTE}

1. Since there were only a small number of trials on which participants made errors, there were limited numbers of data points for the error trials. We therefore used the median rather than the mean to reduce the effects of outliers. However, the same trends were observed when means rather than medians were used.

(Manuscript received October 30, 2006; revision accepted for publication February 1, 2007.) 